\title{
Dietas adelgazantes
}

\section{Slimming diets}

\author{
I. Marques-Lopes ${ }^{1}$, G. Russolillo' ${ }^{1}$, E. Lopes-Rosado' ${ }^{1}$, J. Bressan ${ }^{2}$
}

\section{RESUMEN}

La intervención dietética tiene como objetivos: alcanzar un balance energético negativo, conseguir un peso saludable en la persona obesa y lograr la instauración de hábitos alimentarios saludables para mantener el peso perdido a largo plazo. Considerando el tratamiento dietético de la obesidad un proceso que se manmiento dierécicio mantiene por largos periodos detiempo, la intervención dietética deberá per realizada en función de las características fisiopatológicas y psicosociales de la persona obesa. Además, la terapia dietética deberá ser equilibrada, saludable y adaptada a las preferencias alimentarias del paciente. A lo largo de los años, debido a la necesidad y búsqueda de una pérdida de peso rápida, se han originado, creado e inventado dietas mágicas que se han hecho populares y que carecen de eficacia comprobada y rigor científico. Este trabajo, hace referencia a la dieta hipocalórica equilibrada y revisa las d a la dieta hipocalorica equilibrada y revisa las dietas población obesa y/o con sobrepeso.
pola a cabo por la

Palabras clave: Obesidad. Dieta hipocalórica equilibrada. Dietas mágicas o populares.

\begin{abstract}
Dietetic intervention has the following aims: to achieve a negative energy balance, obtain a healthy weight in the obese person and achieve the installation of healthy eating habits in order to maintain weight loss in the long term. Considering dietetic treatment of obesity as a process that is maintained for long periods of time, dietetic intervention should be carried out depending on the physio-pathological and psycho-social characteristics of the obese person. Besides, dietetic therapy should be balanced, healthy and adapted to the eating preferences of the patient. Over the years, due to need and the quest for rapid weight loss, magic diets have been occasioned, created and invented that have become popular and that lack proven efficacy and scientific rigour. This paper makes reference to a balanced hypocaloric diet and reviews the slimming diets that are best known and most used by the obese and/or overweight population.
\end{abstract}

Key words: Obesity. Balanced hypocaloric diet. Magic or popular diets.

ANALES Sis San Navarra 2002; 25 (Supl. 1): 163-173.

1. Departamento de Fisiología y Nutrición. Universidad de Navarra

2. Departamento de Nutrição e Sanide. Universidad Federal de Viçosa. Brasil

\section{Correspondencia:}

Iva Marques-Lopes

Departamento de Fisiología y Nutrición

Universidad de Navarra

C/ Irunlarrea, s/n

31080 Pamplona

Tfno. 948425600 (ext. 6439)

Fax 948425649 


\section{INTRODUCCIÓN}

La definición de dieta según el diccionario de la Real Academia Española es "Régimen que se manda observar a los enfermos o convalecientes en el comer y en el beber". La sabiduría popular hace alusión a la relación entre la obesidad con enfermedad y el ayuno como forma de curación. Así, por ejemplo el refrán "Para quien con muchos manjares no faltan enfermedades o quien comió hasta enfermar ayune hasta sanar" lleva implícita la idea de que el paciente obeso ingiere alimentos en exceso y es reacio a la restricción energética ${ }^{1}$.

A pesar de los grandes avances en la comprensión de la génesis de la obesidad en sus aspectos genéticos, neuroendocrinos y metabólicos, la sobreingesta y/o el desequilibrio en el balance energético, sigue siendo el principal factor en el origen del aumento de grasa corporal habiéndose descrito que el $90-95 \%$ de los pacientes que pierden peso, lo recuperan al final de varios años $^{2,3}$.

\section{TRATAMIENTO DIETÉTICO DE OBESIDAD}

Los fundamentos de la dieta en el tratamiento de la obesidad están bien establecidos, al menos desde el punto de vista teórico. Con el tratamiento dietético se pretende alcanzar un balance energético negativo para que el organismo obtenga energía para su funcionamiento a través de los depósitos grasos, que constituyen la mayor reserva energética corporal ${ }^{3}$.

Los cambios en la composición corporal derivados del aumento de peso en la obesidad se caracterizan por un aumento de la energía almacenada en forma de grasa que representa aproximadamente el $75 \%$ del peso ganado, mientras que el $25 \%$ del aumento ponderal está representado por la masa libre de grasa ${ }^{4}$. Así, es de gran importancia que el tratamiento dietético seguro en la obesidad se deba enfocar en una pérdida de peso de la misma magnitud y composición que el peso ganado ${ }^{5}$.

El tratamiento dietético de la obesidad se caracteriza no sólo por la implantación de un régimen dietético sino también por la modificación de los hábitos alimentarios y estilo de vida, que incluyen cambios en la actividad física diaria, situaciones de sobreingesta puntual, ingesta impulsiva, o no planeada, así como cambios en la composición de los macronutrientes de la dieta, como por ejemplo la disminución del consumo de lípidos ${ }^{6.8}$.

El tratamiento dietético además de pretender alcanzar un balance energético negativo para la disminución de la masa grasa, también pretende el mantenimiento de la pérdida de peso lograda y prevenir una futura ganancia de peso corporal, siendo estos tres puntos los pilares de la terapia dietética en la obesidad. Sin embargo, si es difícil lograr el primer objetivo, aún es más difícil lograr el segundo y el tercero $^{3}$. Además, la terapia dietética tiene otros objetivos secundarios como son"

- Disminuir los factores de riesgo metabólicos y cardiovasculares.

- Modificar los hábitos alimentarios perjudiciales a la salud.

- Evitar oscilaciones de peso.

- Restablecer el equilibrio psicosomático.

- Mejorar la capacidad funcional y la calidad de vida.

La valoración de la motivación del paciente obeso para la pérdida de peso es fundamental para que el tratamiento sea eficaz, donde no sólo es importante el empeño del profesional de salud, sino también del paciente.

Existen además de la motivación del paciente otros requisitos previos necesarios a tener en cuenta a la hora de iniciar una terapia dietética como son':

- Historia previa de éxitos y fracaso en la pérdida de peso.

- Soporte familiar y de amigos.

- Conocimiento de la importancia y gravedad de la obesidad y de sus comorbilidades asociadas.

- Actitud del paciente hacia el ejercicio físico.

- Obstáculos que el paciente pueda poner a la terapia dietética.

- Consideraciones socioeconómicas del paciente. 


\section{RESTRICCIÓN ENERGÉTICA EN LA OBESIDAD}

Conocida la ingesta del paciente, se plantea el grado de restricción energética de la dieta a realizar. Para ello, es necesario tener en cuenta el peso que se desea alcanzar y el ritmo de adelgazamiento, los cuales dependen de varios factores como son: la edad, enfermedades asociadas y otras condiciones individuales ${ }^{10}$.

Ya que el tratamiento de la obesidad es un proceso crónico, la dieta prescrita deberá poder ser mantenida a largo plazo. Para ello, además de cumplir los requisitos necesarios a la creación de un balance energético negativo, deberá ser nutricionalmente adecuada y seguible por el paciente. Para asegurar un cumplimiento a largo plazo de la dieta hipocalórica prescrita, ésta tiene que ser equilibrada, variada y a ser posible sabrosa y adaptada a las necesidades del enfermo, lo que es lo mismo, individualizada no sólo en cuanto a la restricción energética sino también en cuanto a su composición ${ }^{9-12}$.

Aunque los objetivos de la pérdida de peso se deban individualizar, por norma general se debe pretender una pérdida de peso inicial (primera fase) de alrededor del 10\% del peso corporal al inicio del tratamiento. El ritmo deseable de esa pérdida de peso se sitúa entre $0,5-1 \mathrm{Kg}$ por semana, aunque durante el primer mes se pueda producir una pérdida superior, toda vez que parte del peso inicialmente perdido esta constituido por glucógeno y agua ${ }^{1,3,4}$.

Las pérdidas de peso rápidas no son recomendables, ya que inducen a una pérdida superior de masa magra. Por otro lado, la pérdida de peso excesivamente lenta tampoco es recomendable teniendo en cuenta la posible desmoralización del paciente. Para alcanzar estos objetivos, la ingesta diaria del paciente obeso deberá estar restringida entre 500-1.000 kcal con respecto a la ingesta anterior al tratamiento. Una situación de obesidad mórbida, acompañada de complicaciones como apnea obstructiva o cuadro restrictivo ventilatorio grave, necesita una reducción de peso rápida por su riesgo a corto plazo. Por otro lado, una obesidad moderada sin complicaciones o con complicaciones metabólicas es candidata a una pérdida de peso más progresiva que incida fundamentalmente en una alteración de los hábitos alimentarios a largo plazo. Teniendo en cuenta estos criterios se puede estable$\operatorname{cer}^{5,13}$ :

- Una dieta normocalórica para pacientes con sobrepeso muy leve y con ingesta muy elevada previa al tratamiento.

- Una dieta hipocalórica.

\section{DIETAS HIPOCALÓRICAS}

Las dietas hipocalóricas son aquellas que permiten alcanzar un balance energético negativo, pero que tienen que seguir suministrando igual cantidad de micronutrientes y cumplir los criterios de calidad nutricional exigibles a cualquiera otra dieta $^{3}$.

El tiempo que el paciente mantiene con cada tipo de dieta es variable; un mismo paciente puede pasar también por distintos tipos de dieta, dependiendo de la cantidad de peso a perder y el tiempo necesario para ello.

Las dietas hipocalóricas se dividen en distintos tipos, dependiendo de la restricción energética aplicada ${ }^{1,13,14}$ :

- Dietas con restricción calórica leve: aportan al menos $1.200 \mathrm{kcal} /$ día, que están indicadas en todo paciente con sobrepeso u obesidad que debe perder peso de modo gradual.

- Dietas con restricción moderada (con más de $800 \mathrm{kcal}$ ): debe aportar entre 1.000-1.400 kcal, siendo equilibrada y variada en su composición.

- Dietas con restricción severa que aportan entre 800-1.200 kcal/día. Están indicadas en aquellos pacientes que no responden de modo adecuado a las dietas de restricción calórica moderada y/o que necesitan bajar de peso de modo rápido. Es difícil que puedan cubrir las recomendaciones de vitaminas y minerales y que se ajustan a los hábitos alimentarios del obeso acostumbrado a ingerir volúmenes considerables de distintos alimentos.

- Dietas de muy bajo contenido calórico (VLCD), que habitualmente aportan 
entre 400 y $800 \mathrm{kcal}$. El término se refiere generalmente a las fórmulas comerciales que aportan además, los requerimientos mínimos diarios de vitaminas y minerales. Si estas dietas se obtienen a partir de alimentos naturales, se torna necesaria la utilización de suplementos vitamínicos y minerales.

Las VLCD se utilizan en casos de obesidades mórbidas. Requieren vigilancia estrecha e incluso en muchos casos la hospitalización. En este tipo de dietas los alimentos están muy limitados y el aporte vitamínico y mineral es insuficiente. Puede tener importantes complicaciones, como son la instalación de un desequilibrio hidroelectrolítico, hiperuricemias, deshidratación, hipotensión ortostática, dependiendo del grado de obesidad del paciente y de la situación fisiopatológica en el momento del tratamiento. Este tipo de dieta se recomienda por plazos no superiores a 3-4 meses ${ }^{15}$.

\section{CARACTERÍSTICAS DE LA DIETA HIPOCALÓRICA EQUILIBRADA}

En la primera fase del tratamiento dietético, la composición de la dieta no tiene un efecto significativo en la cantidad o velocidad de la pérdida de peso, bajo condiciones de un balance calórico negativo. Así, el factor que determina de manera más significativa la cantidad y la velocidad de pérdida de peso es el grado de balance energético negativo impuesto por la dieta. Esto se debe a que la pérdida inicial de peso se hace a expensas de masa grasa y masa magra y el efecto de la composición de la dieta en el tejido perdido es mínima. Durante esta fase del tratamiento, las consecuencias más significativas de la composición de la dieta son el efecto termogénico de los alimentos y sobre el grado de adherencia al régimen prescrito. También, la distribución de macronutrientes puede afectar a la sensación de hambre y tener influencia en el grado de cumplimiento de la dieta ${ }^{3,16}$. En las fases posteriores del tratamiento dietético, la composición de los macronutrientes puede influir en la mezcla de sustratos utilizados y en el balance de nutrientes, toda vez que la regulación metabólica es distinta para cada nutriente ${ }^{17}$.

\section{Proteínas}

Las recomendaciones diarias para un adulto normal son de $0,7-0,8 \mathrm{~g} / \mathrm{kg} /$ día. Sin embargo, con una dieta hipocalórica se recomienda $1 \mathrm{~g} / \mathrm{kg} /$ peso de proteínas de alto valor biológico, lo que supone entre 15 a 20\% del valor energético total.

Las recomendaciones dietéticas en cuanto a alimentos proteicos incluyen aquellos de menor contenido lipídico como el pescado blanco, carnes blancas, lácteos descremados y legumbres ${ }^{1,9}$.

\section{Hidratos de carbono}

El aporte de hidratos de carbono debe constituir el 50 ó $60 \%$ de las calorías totales. Se recomienda restringir los azúcares solubles, como la sacarosa y consumir hidratos de carbono ricos en almidón y fibras para lo cual se debe aumentar en proporción el consumo de pan, arroz, pastas, verduras, hortalizas, patatas y legumbres $^{9,18}$. Estos alimentos son ricos en hidratos de carbono complejos, y van acompañados de una cantidad elevada de fibra dietética. Esta tiene un importante efecto saciante, lleva más tiempo para comerlos y además ayudan a prevenir el estreñimiento y parece tener un papel beneficioso sobre el metabolismo lipídico ${ }^{1}$.

Actualmente, la idea de que los hidratos de carbono son promotores del sobrepeso puede ser dejada de parte. Esto se debe a que la capacidad del organismo para almacenar los hidratos de carbono es limitada y está bien controlada, así la oxidación de hidratos de carbono se ajusta a la cantidad ingerida, a diferencia de la grasa cuya regulación metabólica es deficiente $^{17}$.

\section{Lípidos}

El contenido graso de una dieta hipocalórica equilibrada no debe sobrepasar el $30 \%$ de la energía total, incluyendo en este porcentaje un consumo prioritario de aceite de oliva, por su riqueza en componentes antioxidantes y su conocido efecto benefi- 
cioso en la hipertensión arterial, perfil lipídico y glucémico en pacientes obesos ${ }^{1,9}$.

El elevado número de estudios epidemiológicos evidencian que la alta representación de grasas en la dieta promueve el desarrollo de obesidad. La importancia de la grasa en el aumento de grasa corporal adviene de dos aspectos fundamenta$\operatorname{les}^{8,19}$ :

- Se ha visto que dietas ricas en grasa inducen a un aumento del consumo de alimentos superior a las dietas ricas en hidratos de carbono, debido principalmente al poco efecto saciante, densidad calórica de los alimentos ricos en grasa, y procesamiento postabsortivo.

- El segundo aspecto se refiere a la relación existente entre una situación de balance energético positivo y el balance graso, ya que al contrario de las proteínas y hidratos de carbono, la regulación metabólica de los lípidos no es tan eficiente, siendo utilizada o almacenada en respuesta a las variaciones diarias del balance energético.

\section{Vitaminas y minerales}

Las vitaminas, minerales y oligoelementos son constituyentes imprescindibles de una dieta equilibrada, debiendo ser administrados durante un régimen hipocalórico en la obesidad. El suministro de estos elementos se torna deficiente cuando el aporte calórico es inferior a $1.200 \mathrm{kcal}$, por lo que puede ser aconsejable la suplementación vitamínica y mineral en dietas inferiores a $1.200 \mathrm{kcal}$, mantenidas por largos períodos de tiempo ${ }^{1}$.

\section{DIETAS MÁGICAS O POPULARES}

A lo largo de los años se han desarrollado o incluso se han inventado diversos tipos de dietas populares para conseguir una pérdida de peso rápida e eficaz que en muchos casos es fruto de una búsqueda de beneficios económicos, más que la promoción de una dieta sana e equilibrada.

Estas dietas o combinaciones de alimentos que prometen la solución para la pérdida de peso están basadas en principios científicos infundados o en el mejor de los casos no demostrados científica- mente $^{20}$. El uso terapéutico de las denominadas "dietas milagrosas" carece de validez científica en el tratamiento de la obesidad, ya que en la mayoría de los casos, pacientes obesos que se han sometido a estas dietas de moda, a la pérdida de peso le sigue una recuperación ponderal mayor que el adelgazamiento obtenido, y en el peor de los casos, carencias nutricionales y alteraciones metabólicas que pueden conducir a enfermedades severas e incluso hasta la muerte.

\section{Aspectos comunes}

La mayoría de las dietas mágicas poseen algunos aspectos en común ${ }^{21}$ :

- Están prescritas por personas ajenas al campo de la nutrición.

- Tienen una descripción muy imprecisa.

- Auguran elevadas promesas de pérdida de peso para la motivación del paciente.

- No poseen eficacia comprobada.

- Poseen refutables fundamentos dietéticos.

Las "dietas milagrosas" destinadas a tratar la obesidad aparecen en épocas del año como la primavera y verano. Las revistas y semanarios ocupan la mayor parte de su redacción a temas relacionados con dietas de adelgazamiento, incluso se publican monográficos en los que se atrae la atención del consumidor.

La diferencia de llevar a cabo una "dieta milagrosa" o una dieta hipocalórica equilibrada avalada por un experto en nutrición es muy clara: con las dietas milagrosas existe la posibilidad de poner en riesgo la salud. Por tanto, es muy importante aprender a identificar este tipo de dieta y conocer el peligro de llevarlas a cabo.

\section{Clasificación}

La clasificación de las dietas mágicas puede hacerse desde múltiples y variados puntos de vista, según las características de sus componentes, efecto conseguido, procedencia, composición o fundamento al que atribuyen su eficacia ${ }^{20}$. Se pueden encontrar ${ }^{21-23}$ : 
Dietas hipocalóricas desequilibradas: en estas se incluyen la dieta de la Clínica Mayo, Dieta "toma la mitad", Dieta Gourmet, Dieta Cero. Estas dietas provocan un efecto rebote que se traduce en un aumento de masa grasa y pérdida de masa muscular, existiendo una adaptación metabólica a la disminución drástica de la ingesta energética, que se caracteriza fundamentalmente por una disminución del gasto energético. Estos regímenes suelen ser monótonos, además de presentar numerosos déficits en nutrientes sobre todo si se prolongan por largos períodos de tiempo.

Dietas disociativas: Dieta de Hay o Disociada, Régimen de Shelton, Dieta Hollywood, Dieta de Montignac, Antidieta, etc. Se basan en el fundamento de que los alimentos no contribuyen al aumento de peso por sí mismos, sino al consumirse según determinadas combinaciones. No limitan la ingesta de alimentos energéticos sino que pretenden impedir su aprovechamiento como sustrato energético con la disociación.

Dietas excluyentes: se basan en eliminar de la dieta algún nutriente. Estas dietas pueden ser: $i$ ) ricas en hidratos de carbono y sin lípidos y proteínas, como la Dieta Dr. Prittikin y la Dieta del Dr. Haas; $i i)$ ricas en proteínas y sin hidratos de carbono: Dieta de Scardale, Dieta de los Astronautas, Dieta de Hollywood y la Dieta de la Proteína Líquida. Producen una sobrecarga renal y hepática muy importante; iii) ricas en grasa: Dieta de Atkins, Dieta de Lutz. Se conocen como dietas cetogénicas. Pueden ser muy peligrosas para la salud, produciendo graves alteraciones en el metabolismo (acidosis, cetosis, aumento de colesterol sanguíneo, etc.).

\section{Descripción}

\section{Dieta de la Clínica Mayo}

La dieta de la Clínica Mayo ha sido famosa durante muchos años y a pesar del nombre referente a la Clínica Mayo, esta institución no se identifica con este régimen dietético. Consiste en seguir una dieta en la que se elimina el consumo de productos lácteos y se consumen huevos, carnes y guarniciones de verdura. Las bebidas permitidas son el té o el café. Esta dieta aporta entre 600 y $800 \mathrm{kcal}$ y reduce de manera importante la ingesta de calcio dietético ${ }^{21}$.

\section{Dieta disociada de Hay}

La Dieta Disociada o separada de Hay tuvo su inicio entre los años 1900 y 1920. Aunque no es la dieta mágica más peligrosa, es la dieta más famosa realizada en estos últimos años. Sostiene la teoría de que los hidratos de carbono no pueden ser consumidos junto con las proteínas, ya que las proteínas se digieren en medio ácido y los hidratos de carbono en medio alcalino. En principio, este tipo de ingesta es casi imposible, porque no existen alimentos que solamente contengan proteínas o hidratos de carbono. Esta dieta carece de fundamento científico y los resultados obtenidos se deben a una menor ingesta de energía.

Este régimen dietético conlleva fundamentalmente a una pérdida progresiva de la motivación para ingerir alimentos, ya que cada día al paciente sólo le está permitido la ingesta de un solo alimento, aunque en cantidades elevadas ${ }^{20}$.

\section{Dieta de Atkins}

Este tipo de dieta pertenece al grupo de dietas milagrosas llamadas científicamente "dietas cetógenas". En las dietas cetógenas se retira absolutamente el consumo de hidratos de carbono y se potencia el consumo de proteínas y grasas. Como se ha dicho anteriormente, el consumo de hidratos de carbono es la principal fuente de energía del organismo, es el primordial sustrato energético. Cuando el organismo no dispone de este nutriente para obtener energía empieza a quemar las grasas por una ruta metabólica muy particular, produciendo cuerpos cetónicos, que se utilizarán como moneda energética a falta de hidratos de carbono. El resultado es el aumento en sangre de cuerpos cetónicos y sus productos de desecho, entre ellos la acetona ${ }^{21,23}$. En esta dieta, se prohíbe el consumo de leche, frutas, casi todas las verduras, pan, pasta, cereales, arroz, féculas, legumbres, azúcar, dulces, etc. Sólo se pueden tomar carnes, pescados, 
huevos, embutidos, algunos quesos, café, e incluso se permite la toma de grasas, aceites, vísceras, mariscos y en algunas ocasiones alcohol. Este tipo de dieta provoca la falta de apetito, cetosis en sangre, halitosis o acetona en aliento, y en algunas situaciones, riesgo de afección cardiovascular por exceso de consumo de grasas o sobrecarga en la función renal por la exagerada ingesta de proteínas. Este tipo de dieta conlleva una rápida pérdida de peso durante los primeros días, debido a la pérdida de glucógeno utilizado como sustrato energético y agua. El seguimiento de este régimen conduce a una disminución acentuada del apetito, por lo que el paciente disminuye significativamente su ingesta alimentaria. Además del desequilibrio en el metabolismo glucídico, esta dieta produce otras alteraciones donde se destacan también el aumento del colesterol plasmático, debido a la elevada ingesta y disponibilidad hepática y plasmática de lípidos; aumento del ácido úrico plasmático debido al aumento del catabolismo proteico para la obtención de energía ${ }^{23}$.

El rápido aumento de peso que se produce al interrumpir este tipo de dieta, se debe fundamentalmente a la reposición de los niveles de glucógeno y agua previos al inicio del tratamiento. En la última revisión científica a las distintas dieta populares ${ }^{20}$, incluyendo la Dieta del Dr. Atkins, se revisan los estudios realizados de forma a validar científicamente esta dieta, concluyéndose que los estudios llevados a cabo fueron de poca duración, donde participaron pocos pacientes, carecían de grupos control, y eran dietas no definidas.

\section{Dieta de Montignac}

La Dieta de Montignac está basada en la prohibición del consumo simultáneo de alimentos que aportan hidratos de carbono y alimentos que aportan grasas. De la dieta se eliminan todos aquellos alimentos que contienen estos dos nutrientes, como es el caso de la leche entera o semientera. La dieta prohíbe el consumo de azúcar, el pan (excepto el integral), patatas, pastas y arroz, alimentos considerados como un veneno. En cuanto a la fruta, sólo se permite consumirla sola y antes de las comi- das para evitar una fermentación en el intestino, afirmación que todavía hoy los científicos insisten que es falsa ${ }^{24}$. Los principios que acogen a este régimen están completamente errados, como el resto de las dietas, la de Montignac no deja de ser en último término una dieta hipocalórica y se fundamentan en criterios tan pintorescos como que las calorías no cuentan. Es una dieta muy semejante a la Dieta de Atkins, con la diferencia que en la de Montignac, está permitido el consumo de bebidas alcohólicas ${ }^{25}$.

\section{Dieta de Haas}

En esta dieta el $80 \%$ de toda la ingesta debe proceder principalmente de hidratos de carbono (cereales, legumbres, hortalizas y frutas), reduciendo el consumo de alimentos que aporten proteínas. Esta dieta puede ocasionar carencias en vitaminas liposolubles y proteínas. El resultado es la pérdida de peso por ser una dieta hipocalórica en la que tras su abandono, la recuperación ponderal es inmediata ${ }^{25}$.

\section{Dieta toma la mitad}

Basada en ingerir la mitad de los alimentos que habitualmente se comen. Desde luego que se pierde peso, pero también se reduce a la mitad la ingesta de nutrientes esenciales para mantener un buen estado de salud ${ }^{21}$.

\section{La cura de Waerland}

Se caracteriza por consumir agua fresca de manantial, patatas, apio, semillas de lino y hortalizas exclusivamente. Esta dieta consiste en una cura de hambre muy drástica y el consumo calórico total no supera las $600 \mathrm{kcal} / \mathrm{día}$. Una vez finalizada la dieta la ganancia de peso está garantizada, especialmente en la persona obesa ${ }^{22}$.

\section{Dieta disociada de Hay. Régimen de Antoine}

Consiste en comer cada día de uno de los grupos de alimentos, es decir, los lunes sólo carnes, los martes sólo lácteos, los miércoles sólo fruta, los jueves sólo huevos, etc. El régimen se realiza durante una semana y se repite una vez al mes. Los 
resultados prometidos son de una pérdida del orden de 4-5 kg por semana ${ }^{20,25}$.

\section{Dieta del ejército israelí}

Consiste en tomar sólo un tipo de alimento durante dos días seguidos, por lo que sobrevivir con este tipo de dieta puede resultar toda una hazaña en la vida de la persona que decida llevarla a cabo. Los resultados son la pérdida de peso, y como en el resto de este tipo de dieta, la ganancia de peso posterior al abandono de la dieta es alarmante ${ }^{22}$.

\section{La antidieta}

Resulta curioso que los inventores de esta dieta, no sólo se conformasen con separar el consumo de alimentos y fomentar una dieta desequilibrada, sino también sostener que la hora del día influye positiva o negativamente en la ingesta del alimento. De esta manera, en la antidieta importa el tipo de alimento consumido y la hora de la toma. En este caso, los hidratos de carbono y las proteínas no se pueden consumir de forma conjunta, se debe consumir muy poca grasa, se establecen horarios para tomar los diferentes tipos de alimentos, incluye alimentos que elimina toxinas del cuerpo; considera el vinagre un veneno que fermenta las digestiones asî como el pan blanco, el azúcar y la leche ${ }^{24,25}$.

\section{Dieta de Rafaella Carra}

La autora de este libro, artista muy conocida, sostiene la teoría de no mezclar hidratos de carbono con proteínas, hipótesis ya aportada en anteriores dietas. Además, aporta consejos totalmente dotados de irracionalidad, como que todo lo que se consume antes de las 8 horas de la mañana no engorda y que la fruta debe consumirse dos horas antes o después de las comidas principales para que no engorde ${ }^{24}$.

\section{Régimen de Scardale}

Está basada en la eliminación del consumo de grasas y aumento del consumo de alimentos proteicos magros, frutas y verduras. Por otro lado, prohíbe el consumo de leche, pan, arroz, patatas, dulces, aceite de condimento, alcohol y zumos de frutas. Además, sólo se puede tomar entre horas zanahoria y apio. La dieta se lleva durante catorce días sin hacer ningún tipo de modificación ${ }^{21}$.

\section{Dieta de Hollywood}

Dieta estricta en la que sólo se permite ingerir un máximo de $600 \mathrm{kcal}$ y se retira el consumo de hidratos de carbono. Esta dieta puede producir cetosis, problemas renales y deficiencias en vitaminas y minerales. La Dieta Hollywood, es una dieta llevada a cabo durante unos pocos días, con un aporte calórico inferior a $1.000 \mathrm{kcal} / \mathrm{día}$, a base de alimentos proteicos, por lo que se considera con efectos negativos en la función renal u otras ${ }^{25}$.

\section{Dieta de la proteína líquida}

Esta dieta consiste en tomar una solución de proteína obtenida a partir de piel de vaca (colágeno). En Estados Unidos ocasionó más de 60 muertes debido a causas diversas, aunque se sabe que la digestión de esta proteína destruye aminoácidos esenciales como el triptófano ${ }^{24}$.

\section{Monodietas o dietas de un único alimento}

Dentro de este tipo de dieta se encuentran: la dieta del pepino, dieta de los cacahuetes, dieta del arroz, dieta de la patata, dieta del pollo, dieta del jarabe de arce, dieta del pomelo, dieta del marisco, dieta del melocotón, cura de las uvas, dieta de la piña, dieta del ajo, etc. Todas ellas consisten en tomar un sólo alimento y obtener resultados maravillosos en la pérdida de peso. Constituyen un grupo de dietas milagrosas aburridas, monótonas y en algunos casos peligrosas, ya que la deficiencia en numerosos nutrientes es una clara evidencia. La pérdida de peso se obtiene por consumir menos calorías y convertir la hora de comer en un total aburrimiento ${ }^{20,21,25}$.

\section{La Dieta de los Astronautas}

Es una dieta muy hipocalórica basado en verduras y un poco de carne a la plancha o un huevo. El aporte calórico total de este régimen se sitúa en 400 a $500 \mathrm{kcal}$ al día, muy deficiente en todos los macro y micronutrientes ${ }^{25}$. 


\section{La Dieta del Dr. Prittink}

Es una dieta compuesta por cereales, frutas y verduras que se hizo muy famosa en Estados Unidos. Es una dieta muy deficiente en proteínas de elevado valor biológico y lípidos. Además es deficiente en vitaminas del complejo B, calcio y hierro ${ }^{20,21}$.

\section{Dieta vegetariana}

Es un tipo de dieta no utilizada fundamentalmente en la obesidad, pero también se utiliza para la pérdida de peso. Existen dos tipos de dieta vegetariana, concretamente $^{26}$ :

- La dieta ovo-lácteo-vegetariana, que permite la ingesta de leche y huevos además de todos los productos de origen vegetal. En lo que respecta al suministro diario de hidratos de carbono y lípidos, este tipo de dieta no presenta ningún inconveniente, dado que los alimentos ingeridos en la dieta vegetariana aportan estos dos macronutrientes. En lo que se refiere al contenido proteico, la ingesta de alimentos vegetales conjuntamente con lácteos y huevos puede suministrar diariamente las cantidades recomendadas. Sin embargo, se puede producir anemia perniciosa, además de alteraciones del sistema nervioso. Por otro lado, la dieta ovolácteo-vegetariana, puede producir déficit de hierro, ya que este mineral se encuentra en mayores cantidades en los alimentos de origen animal como las carnes, así como su absorción está favorecida en estos alimentos.

- La dieta estrictamente vegetariana, en que sólo se ingieren alimentos de origen vegetal. Al igual que la dieta ovo-lácteovegetariana, no se producen deficiencias de lípidos e hidratos de carbono. Con relación al aporte proteico, este se podrá realizar de forma correcta si el paciente conoce el contenido proteico de los distintos alimentos de forma a combinarlos para la obtención de proteínas con buena calidad biológica.

\section{Dieta macrobiótica}

La dieta macrobiótica constituye una forma extrema del vegetarianismo. Según esta teoría, los alimentos se clasifican en dos categorías: los alimentos Ying, pasivos, y los alimentos Yang, activos, y en que la salud depende del equilibrio en la ingesta de alimentos Ying y Yang.

El sistema macrobiótico está constituido por diez dietas. Así, la microbiótica se constituye por 5 dietas, con cantidades decrecientes de alimentos de origen animal. Las otras 5 están constituidas exclusivamente por alimentos vegetales, con un aumento progresivo del consumo de cereales frente a las verduras y hortalizas. Este tipo de dietas conlleva a los mismos problemas que las dietas vegetarianas. En las dietas macrobióticas más estrictas, se producen deficiencias de vitaminas $A, D, E, B_{12}$ y de minerales como el calcio y hierro. Además, esta dieta recomienda la disminución del consumo de agua que puede conducir a un estado de deshidratación, con alteraciones del equilibrio electrolítico y función renal ${ }^{27}$.

\section{Dietas psicológicas}

Afrontan los problemas psicológicos y emotivos que tienen muchas personas relacionados con la alimentación. Consiste en la manipulación psicológica, hipnosis, etc., como la Dieta mental, Psicodieta, etc ${ }^{25}$.

\section{Dieta de la Sopa y de la Luna}

Esta dieta también es llamada de Dieta de Adriane Galisteu, artista muy conocida en Brasil, que propone un menú variable de sopas calientes, heladas y dulces, siempre licuadas. Durante una semana, es el único alimento permitido en las 3 comidas, con repeticiones a intervalos de 15 días. Ocurre el adelgazamiento rápido, pero no asegura una cantidad equilibrada de nutrientes al organismo. La Dieta de la Luna recomienda el consumo exclusivo de líquidos durante 24 horas, a cada cambio de luna. Zumos y caldos son los únicos alimentos permitidos, siendo una dieta pobre en nutrientes. El agua facilita los procesos de digestión y excreción. La pérdida de peso depende de los alimentos elegidos ${ }^{28}$.

Dieta de Beverly Hills o Dieta de las

\section{Frutas}

Productos como piña, papayo y sandía pueden ser ingeridos ad libitum. La dieta 
es aburrida y restringe la vida social del individuo. La pérdida de peso es el resultado de la pérdida de agua por la orina. Provocan carencia de vitaminas $\mathrm{A}, \mathrm{D}, \mathrm{E}$ y $\mathrm{K}^{28}$.

\section{Dieta del Biotipo}

Se basa en la ingesta de alimentos indicados para el tipo físico y temperamento, previendo un adelgazamiento de $5 \mathrm{~kg} / \mathrm{mes}$, sin flacidez. De acuerdo con la biotipología, todos los males, incluso la obesidad, pueden ser tratados con la alimentación. Se hace un test con el objetivo de identificar el órgano que se relaciona con la personalidad del individuo y, con el organismo equilibrado, la persona adelgaza con bajas posibilidades de flacidez y además con buena disposición. En esta dieta también se promete la cura de jaquecas, la tensión premenstrual e incluso la depresión. Promueve la pérdida de peso debido al bajo contenido calórico y grandes cantidades de fibras, pero las bajas cantidades de proteínas pueden provocar el agotamiento de las defensas imnunológicas ${ }^{28}$.

\section{Dieta de los Vigilantes del Peso}

No prohíbe ningún alimento, desde que sean respetadas las cantidades establecidas por el método. Permite las combinaciones de carnes, pastas, dulces y panes. Mantiene la proporción entre hidratos de carbono, proteínas y lípidos. Los seguidores de esta dieta son estimulados por las declaraciones de personas que fueron obesas, en reuniones y conferencias. Esta dieta puede ser utilizada por tiempo indeterminado porque ofrece menús equilibrados y todos los nutrientes necesarios. La pérdida de peso es gradual y tiene orientaciones de nutricionistas y de psicólogos ${ }^{28}$.

\section{Dieta del Grupo Sanguíneo}

Fue creada por el médico americano Peter D'Adamo, que defiende la existencia de una alimentación para cada tipo de sangre $(\mathrm{A}, \mathrm{B}, \mathrm{AB}$ y $\mathrm{O})$, sin considerar el factor Rh negativo o positivo. La dieta muestra los alimentos que reducen o aumentan el peso, de acuerdo con una evaluación histórica de cada tipo sanguíneo. Por ejemplo, frutos del mar y espinaca son indicados para individuos del grupo $\mathrm{O}$, legumbres y verduras para aquellos del grupo A, carnes para el grupo $\mathrm{B}$ y leche para el grupo $\mathrm{AB}$. Por lo tanto, no es una dieta equilibrada. No existe una relación científicamente comprobada entre el tipo de sangre y la utilización de tejido graso. La prohibición de alimentos hace con que la dieta esté asociada con sensaciones de hambre y sufrimiento, e induce a la pérdida de masa libre de grasa, en vez de masa grasa ${ }^{28}$.

\section{Dieta de los Puntos}

El valor calórico de los alimentos es convertido en puntos. Las mujeres pueden ingerir 300 puntos por día y los hombres 400. Cada punto equivale a 3,6 calorías y las comidas deben contener hidratos de carbono, lípidos y proteínas. Es equilibrada y puede ser consumida por tiempo indeterminado, pero se torna inadecuada si el individuo no selecciona bien los alimentos, resultando deficiente en nutrientes esenciales ${ }^{28}$.

\section{Dieta de la USP (Universidad de São Paulo)}

Pese a que ha recibido este nombre, la USP no confirma la autoría de la dieta. Una cierta combinación de alimentos debe ser seguida por 2 semanas. Se recomienda el consumo de mucha proteína y casi ningún hidrato de carbono. Jamón, huevos y café solo sin azúcar son los ingredientes principales. Lleva a pérdida rápida de peso, pero no estimula la combinación de los alimentos, siendo carente en vitaminas A, B y C, y conlleva a aumento de los niveles de ácido úrico $^{28}$.

\section{CONCLUSIONES}

Las dietas milagrosas son normalmente dietas sin fundamento, aunque es cierto que en algunas ocasiones se obtienen una parte de los resultados prometidos. Pero no hay que olvidar que la pérdida de peso conseguida es debido a que se consumen menos calorías, la alimentación se torna aburrida, se pierde el apetito y la ilusión de comer y sobre todo, existe un riesgo importante de recuperar el peso perdido de forma alarmante una vez se ha abandonado el tratamiento dietético, junto con desequilibrios y deficiencias nutricionales. El tratamiento 
de la obesidad es un proceso a largo plazo, en que no sólo está implicada la pérdida de peso, sino también la adquisición de hábitos de vida saludables, por lo que deberá ser llevado a cabo por facultativos sanitarios expertos en nutrición equilibrada y alimentación saludable.

\section{BIBLIOGRAFÍA}

1. LEón VM, GARcía LunA PP. La dieta equilibrada en el tratamiento de la obesidad. En: Obesidad: Presente y Futuro. Ed. Moreno B, Monereo S, Álvarez J. Grupo Aula Médica, Madrid 1999: 263-275.

2. WoOley SC, GARNER DM. Dietary treatments of obesity are ineffective. Br Med J 1994; 309: 655-656.

3. VAN GAAL LF. Dietary treatment of obesity. En: Handbook of Obesity. Ed. Bray GA, Bouchard C, James WPT, Marcell Dekker, Inc., New York, 1998: 875-890.

4. GARROW JS. Dietary methods: an overview. En: Body Weight Control. Ed. Beuder A, Brookes LJ. Churchill Livingstone. Nueva York 1987: 109-116.

5. GARRow JS. Should obesity be treated? Treatment is necessary. Br J Med 1994; 309 : 654-655.

6. WAdden TA, Bell ST. Obesity. En: International Handbook of Behaviour Modification and Therapy. Vol. II. Plenum Press. Nueva York, 1990: 449-472.

7. Devlin MJ, Walsh BT, Spizer RL, Hasin D. Is there another binge eating disorder? A review of the literature on overeating in the absence of bulimia nervosa. Int $\mathrm{J}$ Eating Disord 1992; 11: 333-340.

8. MARTínEz JA. Body-weight regulation: causes of obesity. Proc Nutr Soc 2000 59: 337-345.

9. Vázquez C, Del Olmo D, López Del Val T. Tratamiento dietético: Dieta hipocalórica equilibrada. En: Obesidad: Presente y Futuro. Ed. Moreno B, Monereo S, Álvarez J. Grupo Aula Médica. Madrid, 1999: 191-202.

10. BlackBuRN GL. How much weight loss? En: Obesity Research VII. Ed. Angel A. Londres, 1996: 621-625.

11. MADRID CONESA J. El tratamiento incorrecto de la obesidad. En: El libro de la Obesidad y su tratamiento. Juan Madrid Conesa. Arán Ediciones, S.A. Madrid, 1998: 93-109.

12. Bender A. Salud y fraude. Ediciones Labor. Barcelona, 1987.

13. WADDEN TA. Treatment of obesity by moderate and severe caloric restriction. Ann Intern Med 1993; 119: 688-693.
14. Scheen A, Desaive C, Lefebvre P. Therapy for obesity: today and tomorrow. Baillières Clin Endocrinol Metab 1994; 8: 705-727.

15. Coronas R, Forga M, Leyes P. Dietas de muy bajo contenido calórico (VLCD). Nutrición y Obesidad 2000; 3: 300-305.

16. SASTRE A. ¿Es importante el origen de las calorías de la dieta? Formación continuada en Nutrición y Obesidad, 1998; 2: 56-57.

17. MARQues-Lopes I, ANSOREnA D, Astiasaran I, Forga L, Martínez JA. Postprandial de novo lipogenesis and metabolic changes induced by a high-carbohydrate, low-fat meal in lean and overweight men. Am J Clin Nutr 2001; 73 : 253-261.

18. Russolillo G. Protocolo de intervención dietética en la obesidad. Ed. Cursos a Distancia sobre Nutrición y Salud. Universidad de Navarra, 1999.

19. Astrup A, Buemann B, Christensen NJ, Toubro S. Failure of dietary fat intake to promote fat oxidation: a factor favouring the development of obesity. Am J Clin Nutr 1989; 53: 1124-1129.

20. Freedman MR, King J, Kennedy E. Popular diets: A scientific review. Obes Res 2001; 9: 1-40.

21. BENDER A. Dietas mágicas y otros errores. En: Reflexiones sobre Nutrición Humana. Ed. Fundación BBV, 1994.

22. Varela G, Núnez C, Moreiras O, Gran Covian F. Dietas Mágicas. Documento Técnico de Salud Pública №42. Comunidad de Madrid. Ed. Conserjería de Sanidad y Servicios Sociales, 1999.

23. CABO RJ. Peligros de las dietas cetogénicas en el tratamiento de la obesidad. Rev Esp Nutr Comunitaria 1995; 1,4: 110-114.

24. Mataix J. Adelgazar: verdades y falsedades. Ed. Alhulia, 1998.

25. MADRID CJ. Tratamiento dietético incorrecto de la obesidad. En: El libro de la obesidad y su tratamiento. Ed. Arán, Ediciones S.A., Madrid, 1998: 93-110.

26. Ballesteros-Pomar MD, Rubio-Herrera MA, Gutierrez-Fuentes JA, GoMEZ-GeriQue JA, Gomez-De-LA-CAmara A, PASCuAl $\mathrm{O}$ et al. Dietary habits and cardiovascular risk in the Spanish population: the DRECE study (II) micronutrient intake. Dieta y Riesgo de Enfermedades Cardiovasculares en España. Ann Nutr Metab 2000; 44: 177-182.

27. Kennedy ET, Bowman SA, Spence JT, Freedman M, KING J. Popular diets: correlation to health, nutrition, and obesity. J Am Diet Assoc 2001; 101: 411-420.

28. Sachs A, Brazaca SC, MonteIRo JBR. Guia: na selva dos regimes. Época. 2000: 56-59. 